\title{
Radio array starts work
}

\section{Giant low-frequency sensor system on track to probe the birth of the first stars.}

\section{BY ERIC HAND}

$\mathrm{T}$ he Netherlands, one of the most densely populated countries in Europe, would seem to be an inauspicious place to detect radio whispers from the distant Universe. Mobile-phone towers, television transmissions, planes overhead and even the odd burst of noise from a windmill create a background din in the radio sky.

But the builders of LOFAR, the Low-Frequency Array of radio receivers centred around the tiny village of Exloo, say that they have found ways to ignore the noise. In doing so, Dutch astronomers at ASTRON, the Netherlands Institute for Radio Astronomy in Dwingeloo, are opening up a region of the electromagnetic spectrum that should hold clues to one of the earliest phases of cosmic history, when the first stars formed - an era beyond the ken of even the biggest optical telescopes.

"Many of the radio astronomers said this couldn't be done," says Heino Falcke, an astronomer at Radboud University in Nijmegen and chairman of the International LOFAR Telescope Board, the five-nation foundation that governs the $€ 150$-million (US\$192-million) project. Yet Falcke and his colleagues defied the doubters by presenting their first results on 9 January at a meeting of the American Astronomical Society in Austin, Texas. "The message today is: the basic things all work. We can do this," he said.

Mapping the low-frequency sky requires an expansive telescope as well as the ability to tune out noise. When completed later this year, the array will contain 2,700 slender

\section{A MULTI-PURPOSE ARRAY}

The Low Frequency Array was designed for astronomy - but its fibre-optic data links will also be used for seismic monitoring, water management and agricultural sensing.

At the core of the array will be 40 stations centred around the Dutch village of Exloo.

Date stations completed:

- 20092010 - 2011

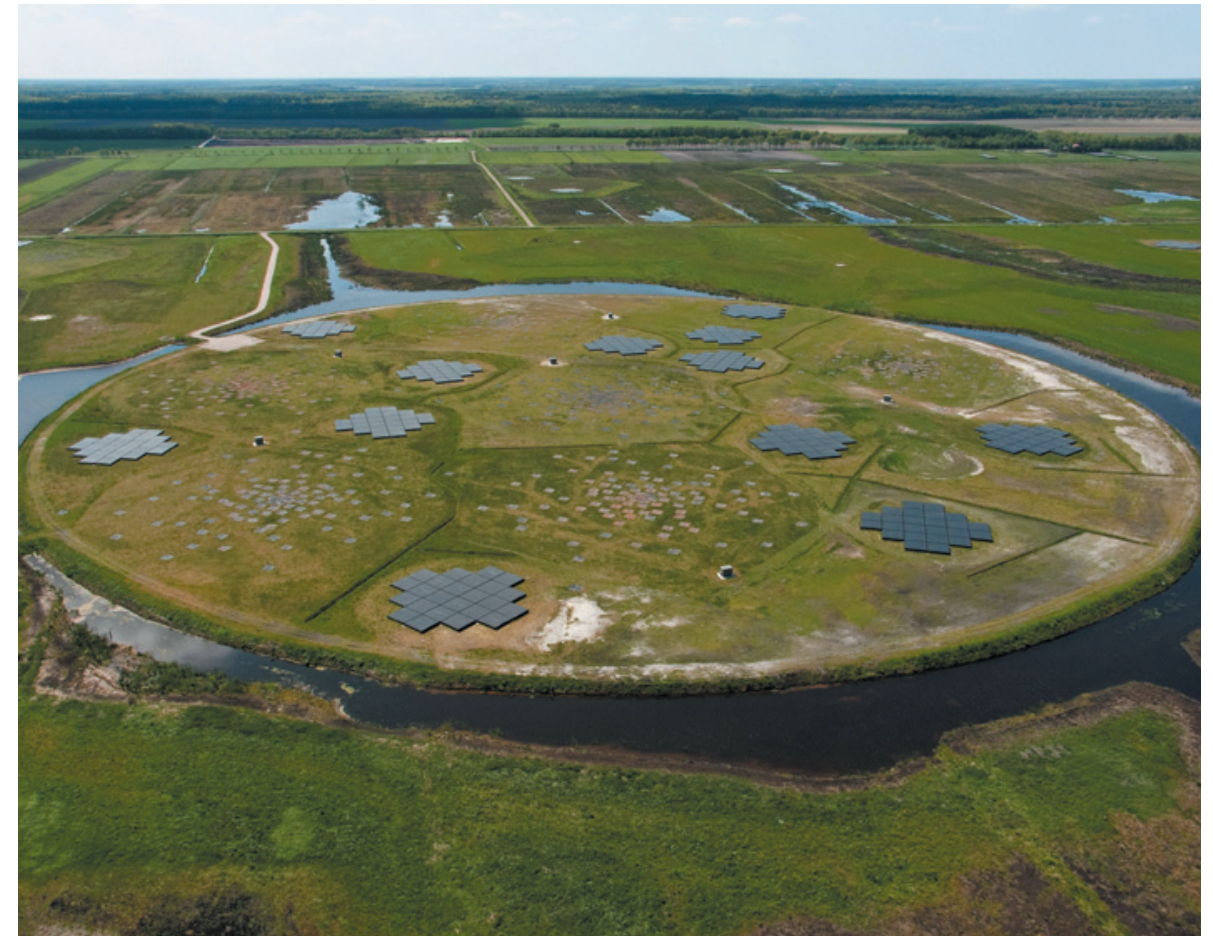

At the heart of the LOFAR telescope is a collection of tile-like and dipole antennas.

dipole antennas tuned to $30-80$ megahertz, and 43,000 antennas embedded in flat tiles a few metres square that are sensitive to 120-240 megahertz. The antennas will be concentrated in 40 stations across the Netherlands; 8 other stations in the United Kingdom, France, Germany and Sweden give the array extra angular resolution for fine-scale imaging. The fibre-optic data network that links the LOFAR antennas will also support sensor webs for geoscience and agricultural monitoring (see 'A multi-purpose array').

LOFAR has already turned up surprises. Its observations of four pulsars - spinning neutron stars that emit regular radio pulses - show that the emissions at a wide range of frequencies are bunched together in time, implying that they emanate from a narrow region near the pulsar. The finding challenges a common picture in which pulsars generate their radio signal in a much wider region, says ASTRON's Jason Hessels, co-principal investigator of LOFAR's pulsar working group.

But the real prize would be the detection of a signal from the epoch of re-ionization. That was roughly 400 million to 800 million years after the Big Bang, when radiation from the first stars and galaxies ionized the primordial hydrogen gas that filled the newborn Universe, creating bubbles of ionized gas like the holes in a Swiss cheese. By mapping the gradual disappearance of the faint 21-centimetre emission line from the primordial hydrogen, LOFAR astronomers hope to distinguish between two re-ionization mechanisms: one driven by the stars' ultraviolet light and one by X-rays from super-massive black holes in newborn galaxies. That would allow the astronomers to watch the first stars and galaxies being born.

Based on an initial run of ten hours spent staring at several quiet corners of the radio sky, ASTRON's Ger de Bruyn suspects that the re-ionization signal could be detected with 400 hours of devoted observing time - a campaign he plans to begin by the end of the year.

That schedule would put LOFAR ahead of rival re-ionization projects in remote parts of Australia, South Africa and New Mexico, locations chosen partly for their lack of radio noise. Greg Taylor, an astronomer at the University of New Mexico in Albuquerque and director of the Long Wavelength Array being built in New Mexico, acknowledges that LOFAR is the array to beat in the race to detect re-ionization - but says that would be just the beginning. "If you make the discovery, then it's a Nobel prize for somebody," he says. But afterwards, "it has the potential to explode into a field of study". 